\title{
Homogenization-based topology optimization for high-resolution manufacturable micro-structures
}

\section{Groen, Jeroen Peter; Sigmund, Ole}

Published in:

International Journal for Numerical Methods in Engineering

Link to article, DOI:

$10.1002 / \mathrm{nme} .5575$

Publication date:

2018

Document Version

Peer reviewed version

Link back to DTU Orbit

Citation (APA):

Groen, J. P., \& Sigmund, O. (2018). Homogenization-based topology optimization for high-resolution manufacturable micro-structures. International Journal for Numerical Methods in Engineering, 113(8), 11481163. https://doi.org/10.1002/nme.5575

\section{General rights}

Copyright and moral rights for the publications made accessible in the public portal are retained by the authors and/or other copyright owners and it is a condition of accessing publications that users recognise and abide by the legal requirements associated with these rights.

- Users may download and print one copy of any publication from the public portal for the purpose of private study or research.

- You may not further distribute the material or use it for any profit-making activity or commercial gain

- You may freely distribute the URL identifying the publication in the public portal

If you believe that this document breaches copyright please contact us providing details, and we will remove access to the work immediately and investigate your claim. 


\title{
Homogenization-based topology optimization for high-resolution manufacturable micro-structures
}

\author{
Jeroen P. Groen ${ }^{\mathrm{a}, 1, *}$, Ole Sigmund ${ }^{\mathrm{a}}$ \\ ${ }^{a}$ Department of Mechanical Engineering, Solid Mechanics, Technical University of Denmark
}

\begin{abstract}
This paper presents a projection method to obtain high-resolution, manufacturable structures from efficient and coarse-scale, homogenization-based topology optimization results. The presented approach bridges coarse and fine scale, such that the complex periodic micro-structures can be represented by a smooth and continuous lattice on the fine mesh. A heuristic methodology allows control of the projected topology, such that a minimum length-scale on both solid and void features is ensured in the final result. Numerical examples show excellent behavior of the method, where performances of the projected designs are almost equal to the homogenization-based solutions. A significant reduction in computational cost is observed compared to conventional topology optimization approaches.
\end{abstract}

Keywords: topology optimization, homogenization, manufacturing constraints, high-resolution

\section{Introduction}

Nowadays, the wide-spread availability of computational resources has resulted in topology optimization as a mature design method, with numerous applications in industry. Nevertheless, large-scale topology optimization still comes at a high computational cost, dominated by the finite element analysis [1]. Furthermore, to ensure near optimal solutions, continuation approaches have to be used which increases the number of design iterations and computational time. Theoretically it is known that that the optimal topology consists of periodic composites with infinitesimally small features. In homogenization-based topology optimization the space of admissible designs is relaxed to allow such composites [2]. In this way, that provided the basis for the original works in topology optimization, optimal solutions could be obtained at a much lower computational cost compared to density-based topology optimization.

Even more than an optimal design, a manufacturable structure is required, which can of-course not consist of infinitesimally small features. To get manufacturable designs out of the relaxed solutions, several multi-scale techniques have been used. Rodrigues et al. proposed a hierarchical optimization scheme which combines free material optimization (FMO) on the macro-scale, with an optimization of the micro-structure using inverse homogenization [3]. This approach was later extended to allow for parallel computations [4], as well as for non-linear elasticity [5]. To reduce

\footnotetext{
${ }^{*}$ Correspondence to: J. P. Groen, Department of Mechanical Engineering, Solid Mechanics, Technical University of Denmark, Nils Koppels Allé, Building 404, 2800 Kgs. Lyngby, Denmark

${ }^{1}$ E-mail: jergro@mek.dtu.dk
} 
the computational cost one can also consider to restrict the amount of unique micro-structures throughout the domain, however, this comes at a reduction of optimality [6, 7]. A limitation of these methods is that the connectivity between neighboring micro-structures is not guaranteed. Greifenstein and Stingl approached this problem by constraining the variation of the material [8]. Multi-scale techniques such as the aforementioned approaches have received rapid growing interest, in part spurred by the increased availability of additive manufacturing (AM) techniques. By far most of the published work (all of which shall not be listed here) is based on "separation of scales", i.e. it is assumed that microscopic patterns are much smaller than the macroscopic details, in turn allowing for separate modelling of the micro and macro scales. In connection with AM one should also mention the treatment of in-fill, i.e. porous structures that, apart from weight saving, also may increase buckling stability of topology optimized structures, c.f. [9, 10].

In a different approach Lazarov and Alexandersen employ the multi-scale finite element method (MsFEM) to design high-resolution manufacturable structures $[11,12]$. These works do not assume separation of scales and hence provide better analysis and design of structures with finite periodicity, although the computational cost is high compared to homogenization-based topology optimization.

In a very appealing approach Pantz and Trabelsi introduced a method to project the microstructures from the relaxed design space to obtain a solid-void design with finite length-scale [13, 14]. The local structure is oriented along the directions of lamination such that a well-connected design is achieved. This approach paves the way for coarse-scale topology optimization where the projection can be performed on a high-resolution mesh in a post-processing step, without a need for cumbersome and expensive multi-scale formulations. In a related study Rumpf and Pazos show that any type of (also spatially varying) unit-cell, represented by a Fourier series, can be projected on a fine scale mesh $[15,16]$.

This paper shall be seen as a simplification and improvement of the approach introduced by Pantz and Trabelsi $[13,14]$. We simplify the projection approach and introduce procedures for controlling the size and shape of the projected design, such that high-resolution (e.g. 1.3 million elements in 2D), near-optimal and manufacturable lattice designs can be achieved within a few minutes in a single processor Matlab code on a standard PC. This short time allows a designer to get high-resolution manufacturable designs at speeds, which potentially can make high-resolution topology optimization an integrated part of interactive design processes [17].

The paper is organized as follows: The methodology of numerical homogenization, and how it can be used in the context of topology optimization is introduced in Section 2. The projection method and its implementation details are presented in Section 3. In Section 4 a method to control the shape of the projected designs is shown. Corresponding numerical examples are shown in Section 5. Finally, the most important conclusions of this study are presented in Section 6.

\section{Homogenization-based topology optimization}

It is well-known that for many topology optimization problems, the optimal solutions can be found in the relaxed design space, i.e. the space allowing for micro-structural materials which have an infinitely fast variation in solid and void regions $[18,19,20]$. At the microscopic scale, these micro-structures are assumed to be uniform, hence they can be represented by periodic unit cells. While at the macroscopic scale geometries and orientations are spatially varying such that optimal structural properties can be obtained. In the original works on topology optimization, micro-structures were either described by square cells with rectangular holes [2], or as layered materials [21]. Similar to these works, we apply topology optimization to compliance minimization 
of linear-elastic plane problems subject to a single load case, where the main focus of this study is on the physical realization of these optimized topologies.

Avellaneda has shown that the optimal solution for minimum compliance problems is in the space of layered materials, the so-called rank-n laminates [20]. Here rank-2 laminates are optimal for plane problems subject to a single load case, and rank-3 laminates are optimal for plane problems subject to multiple load cases. Unfortunately, rank-n laminates require different length-scales, which poses a challenge on their physical realization. Therefore, we restrict ourselves to the sub-optimal (but close in performance) square unit-cells with rectangular holes, c.f. [2], An illustration of one unit cell, with its local coordinate system $\left(\mathrm{y}_{1}, \mathrm{y}_{2}\right)$, and link to the global coordinate system $\left(\mathrm{x}_{1}, \mathrm{x}_{2}\right)$ is seen in Figure 1.
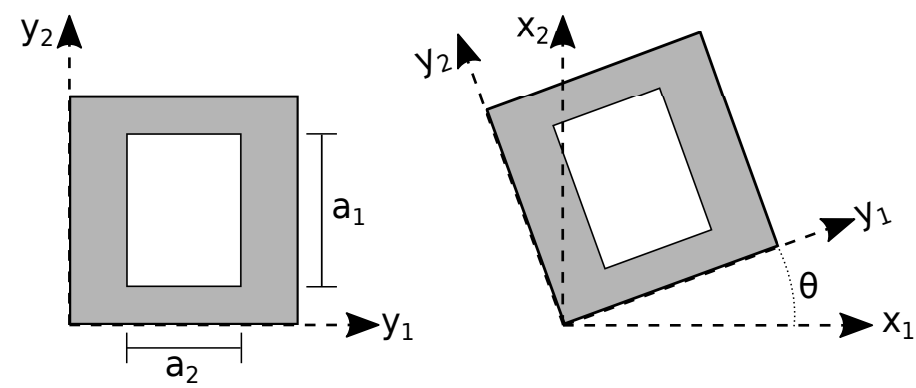

Figure 1: Lay-out of the unit-cell with a rectangular hole, in local $\left(\mathrm{y}_{1}, \mathrm{y}_{2}\right)$, and global $\left(\mathrm{x}_{1}, \mathrm{x}_{2}\right)$ coordinate system

\subsection{Numerical homogenization}

The macroscopic constitutive properties of the unit-cell in its local coordinate system $\left(\mathrm{y}_{1}, \mathrm{y}_{2}\right)$ form the homogenized elasticity tensor $\mathbf{C}^{H}$. These properties, which depend on the parameters describing the height $a_{1}$ and width $a_{2}$ of the hole ${ }^{2}$, can be obtained using numerical homogenization. A discussion on the theory of homogenization is outside the scope of this work, instead the interested readers are referred to $[22,23,24,25]$, in which detailed descriptions of the theory and implementation can be found.

In topology optimization, the parameters $a_{1}$ and $a_{2}$ are spatially varying, and subject to change during each design iteration. Therefore, it is cumbersome to perform numerical homogenization for each variation in $a_{1}$ and $a_{2}$. Instead, we can calculate $\mathbf{C}^{H}$ for a large number of combinations of $a_{1}$ and $a_{2}$, and interpolate between them, as proposed in [2]. To do so, the publicly available and easyto-use MATLAB code by Andreassen and Andreasen is used [26]. With this code, a mesh consisting of $100 \times 100$ bi-linear finite elements has been created, on which $\mathbf{C}^{H}$ has been determined for $51 \times 51$ different combinations of $a_{1}$, and $a_{2}$. The used material properties are $E=1$, and $\nu=0.3$, while a very compliant material plays the role of void, i.e. $E_{\text {void }}=10^{-9} \mathrm{E}$. To avoid discontinuities in elastic properties we treat the hole as an infinitesimally thin crack when one of the parameters $a_{i}$ takes the value 0 . The resulting curves for the 4 unique indices of the homogenized elasticity coefficients are shown in Figure 2, where linear interpolation is applied to obtain values between the data-points.

\footnotetext{
${ }^{2}$ Hence, $a_{1}$ determines the stiffness of the unit-cell in the $y_{1}$-direction, and $a_{2}$ determines the stiffness of the unit-cell in the $y_{2}$-direction
} 

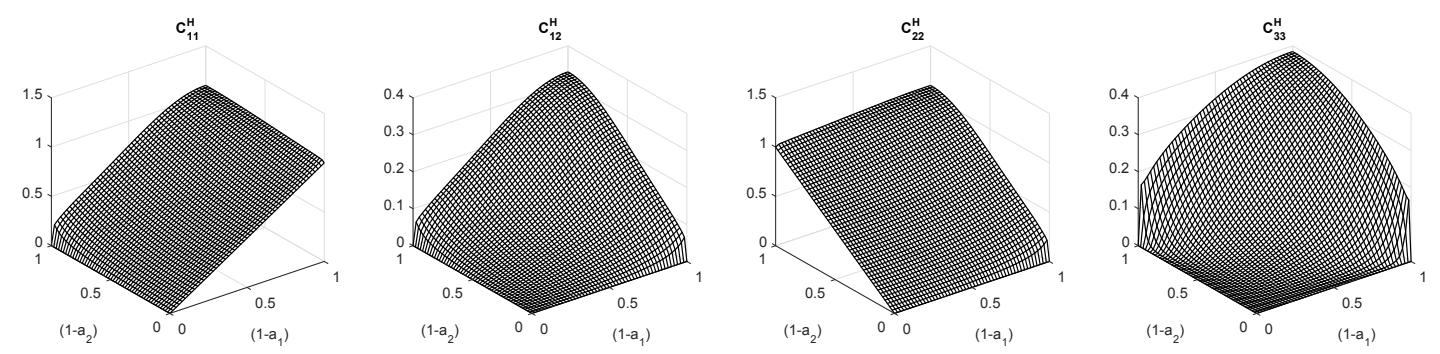

Figure 2: The indices of the homogenized elasticity tensor $\mathbf{C}^{H}$ for different values of $a_{1}$ and $a_{2}$, where $E_{\text {strong }}=1$, and $\nu=0.3$.

With these curves known, the macroscopic elasticity tensor in the global reference frame $\mathbf{C}$ can be calculated.

$$
\mathbf{C}\left(a_{1}, a_{2}, \theta\right)=\mathbf{R}^{T}(\theta) \mathbf{C}^{H}\left(a_{1}, a_{2}\right) \mathbf{R}(\theta)
$$

Where $\mathbf{R}$ is the well-known rotation matrix. Finally, the amount of material in unit cell $m$ can be calculated as,

$$
m=1-a_{1} a_{2}
$$

\subsection{Topology optimization formulation}

Topology optimization is a material distribution problem, where the goal is to find an optimized material distribution that minimizes an objective function, subject to a number of constraints [27]. In this study we will restrict ourselves to minimizing compliance $\mathcal{J}$, for single load-case problems, subject to an upper bound on the volume. The design domain is discretized in elements, in which the shape and orientation of the micro-structure is assumed to be uniform. The local design variables $a_{1}, a_{2}$, and $\theta$, can thus be combined into design vectors $\mathbf{a}_{1}, \mathbf{a}_{2}$, and $\boldsymbol{\theta}$.

The topology optimization problem is solved in nested form, by successive minimizations w.r.t. design variables $\mathbf{a}_{1}, \mathbf{a}_{2}$, and $\boldsymbol{\theta}$, where for each design iteration the equilibrium equations are satisfied by FE-analysis. As is shown by Pedersen [28, 29], the optimal orientation of an orthotropic composite coincides with the principal stress directions, hence $\boldsymbol{\theta}$ is aligned accordingly for each minimization step. Subsequently, design vectors $\mathbf{a}_{1}$ and $\mathbf{a}_{2}$ are updated at each minimization step based on their gradients. The discretized optimization problem can thus be written as,

$$
\begin{aligned}
\min _{\mathbf{a}_{1}, \mathbf{a}_{2}, \boldsymbol{\theta}} & : \mathcal{J}\left(\mathbf{a}_{1}, \mathbf{a}_{2}, \boldsymbol{\theta}, \mathbf{U}\right) \\
\text { s.t. } & : \mathbf{K}\left(\mathbf{a}_{1}, \mathbf{a}_{2}, \boldsymbol{\theta}\right) \mathbf{U}=\mathbf{F} \\
& : \mathbf{v}^{T} \mathbf{m}\left(\mathbf{a}_{1}, \mathbf{a}_{2}\right)-V_{\max } \leq 0 \\
& : \mathbf{0} \leq \mathbf{a}_{1}, \mathbf{a}_{2} \leq \mathbf{1}
\end{aligned}
$$

Where $\mathbf{m}\left(\mathbf{a}_{1}, \mathbf{a}_{2}\right)$ describes the amount of material within an element (c.f. Equation 2), $\mathbf{v}$ is the vector containing the element volumes, and $V_{\max }$ is the maximum allowed volume of the material in the design domain. Stiffness matrix $\mathbf{K}$ is a function of $\mathbf{a}_{1}, \mathbf{a}_{2}$, and $\boldsymbol{\theta}, \mathbf{F}$ describes the loads acting on the domain, and $\mathbf{U}$ describes the solution of the equilibrium equation. For the design update of $\mathbf{a}_{1}$ and $\mathbf{a}_{2}$ the MATLAB implementation of the Method of Moving Asymptotes (MMA) is used [30].

As shown in [31], checkerboard patterns can occur since the strain energy density of these patterns is over-estimated using bi-linear finite elements. To prevent the checkerboarding, the 
solution space is restricted using a density filter applied to $\mathbf{a}_{1}$ and $\mathbf{a}_{2}$ independently [32, 33]. A small filter radius $r_{\min }$ of 1.5 times the element width $h_{c}$ is used, since the filter only has to prevent the occurrence of checkerboard patterns and should not impose a length-scale on the design. The physical dimensions of the hole $\overline{\tilde{\mathbf{a}}}_{1}$, and $\overline{\tilde{\mathbf{a}}}_{2}$ are the filtered design variables. These values are used to determine the homogenized elasticity tensor $\mathbf{C}^{H}$, and to determine the amount of material within a unit cell $m$.

\subsection{Test-problems}

In this study two test problems are considered. The first is a cantilever problem, subject to a distributed unit load over $20 \%$ of the right boundary, shown in Figure 3(a). A volume constraint of 0.5 is set, and a coarse discretization of $80 \times 40$ elements is used to solve the homogenizationbased topology optimization problem. The second problem is a Michell-type problem with circular support, subject to a distributed unit load over 10\% of the right boundary, shown in Figure 3(b). For this problem a volume constraint of 0.25 is set, while a mesh of $80 \times 60$ elements is used to perform the topology optimization. For both problems a solid material with unit stiffness, and a Poisson's ratio of $\nu=0.3$ is used.

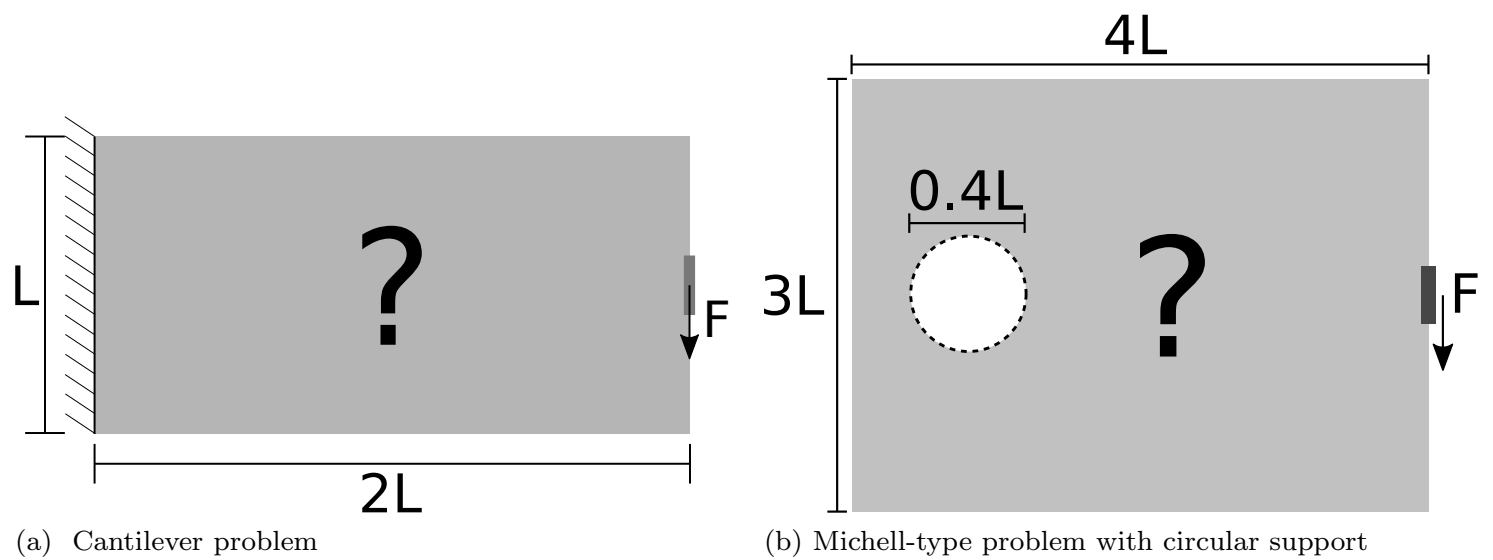

Figure 3: Boundary conditions of the two numerical examples considered in this study

To verify the homogenization-based topology optimization algorithm the cantilever beam problem is solved, which results in a compliance of 58.35. This value is very close to the value of 56.73 obtained using the more optimal rank-2 laminate as micro-structure, reported in [34]. The unit-cell densities of the optimized topology can be seen in Figure 4(a). A close-up of this design can be seen in Figure 4(b), where the red and blue lines correspond to $1-a_{1}$, and $1-a_{2}$ respectively. The challenge is now to convert this result to a practically realizable structure with the highest possible precision using the least computational effort.

\section{Projection of micro-structures on the fine-scale}

Almost any type of periodic micro-structure can be represented by a complex exponential Fourier series with spatially varying parameters $[15,16]$. This allows one to project a complex microstructure on a fine-scale, yet maintaining a smooth and continuous lattice. 


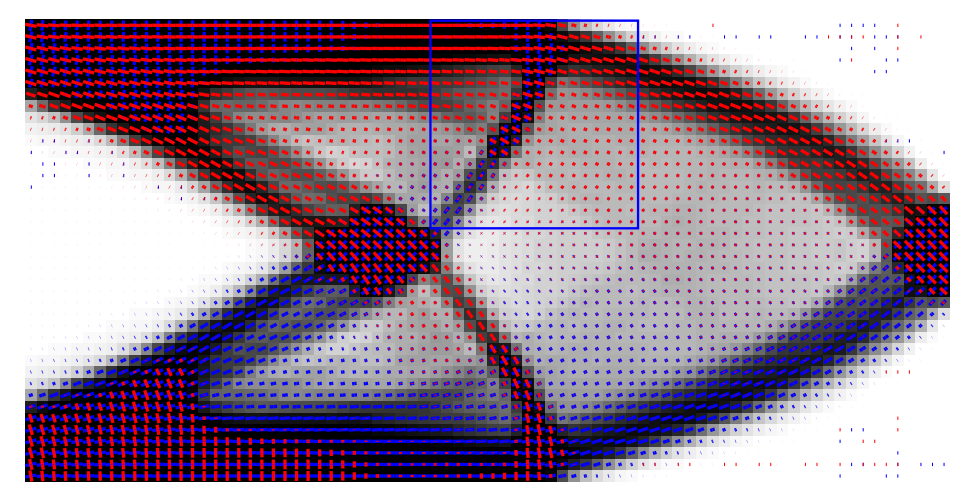

(a) Homogenization-based optimized topology

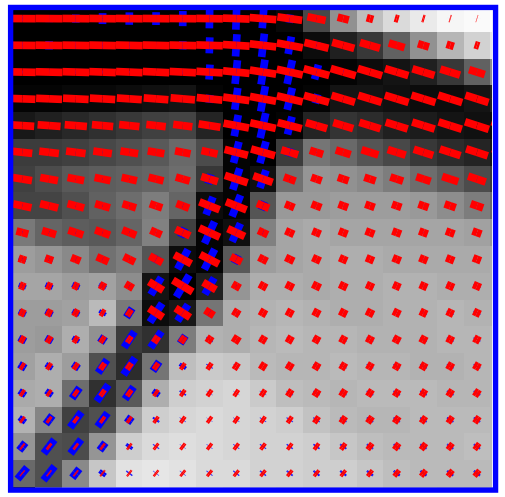

(b) Close-up of the optimized topology

Figure 4: Optimized topology for the $2 \mathrm{D}$ cantilever problem, using $80 \times 40$ elements.

\subsection{Projecting a uniform micro-structure}

The unit cell with a rectangular hole, used in the topology optimization problem, is simple enough to be represented by just two orthogonal cosine waves [13, 14]. The first cosine wave describes the part of the unit cell aligned with $y_{1}$, while the second cosine wave describes the part aligned with $y_{2}$. Each of these cosine waves can be obtained independently from each other, thus we here restrict ourselves to the derivation of the first cosine wave $\tilde{\rho}_{1}$, This cosine wave is oriented using unit-vector $\mathbf{e}_{1}$, such that it is constant in the direction of $y_{1}$, hence $\mathbf{e}_{1}$ is orthogonal to $y_{1}$.

$$
\tilde{\rho}_{1}(\mathbf{x})=\frac{1}{2}+\frac{1}{2} \cos \left(P\left(\mathbf{e}_{1} \cdot \mathbf{x}\right)\right)
$$

Where $\mathbf{e}_{1}$ can be written in terms of the local orientation angle $\theta$ as,

$$
\mathbf{e}_{1}=\left[\begin{array}{c}
-\sin (\theta) \\
\cos (\theta)
\end{array}\right]
$$

$P$ is a term that scales the periodicity based on the size of the unit-cell $\varepsilon$,

$$
P=\frac{2 \pi}{\varepsilon}
$$

Homogenization-based topology optimization is based on an infinite periodicity, i.e. $\varepsilon \rightarrow 0$, however, this can of-course not be realized. Therefore, the micro-structures have to be magnified towards a fine but realizable scale on the macroscopic level, using a finite $\varepsilon$. To take into account the height of the void $\left(a_{1}\right)$, and to get a clear solid-void design, a Heaviside step function is used. Here $\tilde{\rho}_{1}$ is projected to a a physical design $\rho_{1}$, where the threshold parameter $\eta_{1}$ is based on $a_{1}$.

$$
\begin{gathered}
\rho_{1}\left(a_{1}(\mathbf{x})\right)=H\left(\tilde{\rho}_{1}(\mathbf{x})-\eta_{1}\left(a_{1}(\mathbf{x})\right)\right) \\
\eta_{1}\left(a_{1}(\mathbf{x})\right)=\frac{1}{2}+\frac{1}{2} \cos \left(\pi\left(1-a_{1}(\mathbf{x})\right)\right)
\end{gathered}
$$


The shape of the projected unit-cell can then be retrieved using,

$$
\rho(\mathbf{x})=\max \left\{\rho_{1}(\mathbf{x})+\rho_{2}(\mathbf{x}), 1\right\}
$$

Where the second cosine wave $\rho_{2}$, is oriented using unit-vector $\mathbf{e}_{2}$, which is orthogonal to $\mathbf{e}_{1}$. The projection procedure of a sequence of uniform rectangular unit cells can be seen in Figure 5. Figures 5(a) and (b) show cosine waves $\tilde{\rho}_{1}$ and $\tilde{\rho}_{2}$ obtained using Equation 4, while the projected shape of the lattice obtained using Equation 9 is seen in Figure $5(\mathrm{c})$. The domain has unit width and height, with $\varepsilon=0.25$.

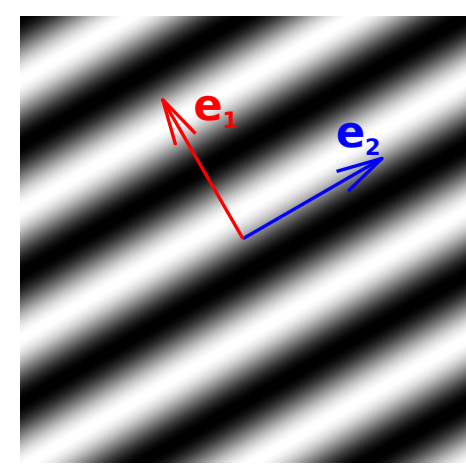

(a) First cosine wave $\tilde{\rho}_{1}(\mathbf{x})$

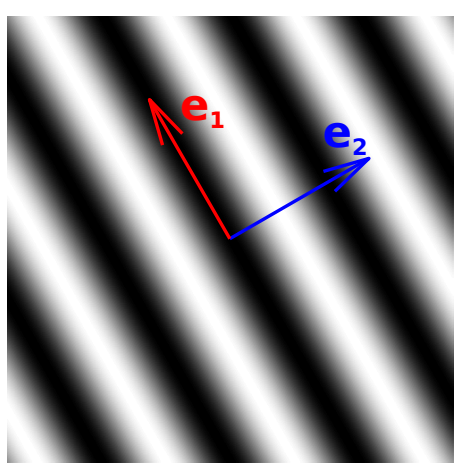

(b) Second cosine wave $\tilde{\rho}_{2}(\mathbf{x})$

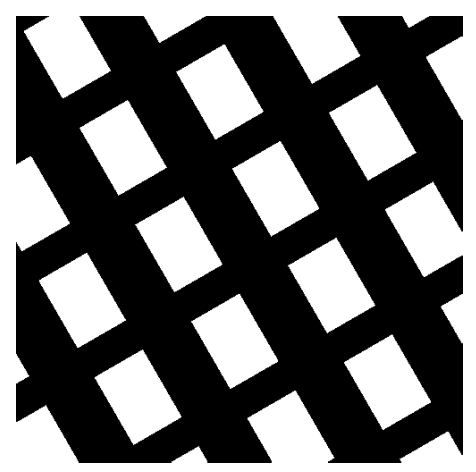

(c) Projected structure $\rho(\mathbf{x})$

Figure 5: Projection of a uniform micro-structure on a fine-scale using just three parameters, $a_{1}=0.7, a_{2}=0.5$, and $\theta=\pi / 6$.

\subsection{Projecting a spatially variant micro-structure}

Equation 4 does not hold when the orientation of the micro-structures is spatially varying. A unit-cell cannot be square if the spatial variation in angles has to be satisfied. Instead, we use a mapping function $\phi_{1}$, which maps the optimization domain $\Omega$ onto a periodic set in $\mathbb{R}^{2}$ that describes the composite, i.e. $\phi_{1}: \Omega \rightarrow \mathbb{R}^{2}[13,14]$. Using this mapping function, we can reformulate Equation 4 such that the cosine wave $\tilde{\rho_{1}}$ can be described by,

$$
\tilde{\rho_{1}}(\mathbf{x})=\frac{1}{2}+\frac{1}{2} \cos \left(P \phi_{1}(\mathbf{x})\right)
$$

The challenge is thus to find a suitable parameterization $\phi_{1}$, such that each point in $\Omega$ corresponds to the correct composite shape. We can solve for $\phi_{1}$ by minimizing the least-squares error between unit vector $\mathbf{e}_{1}$ and the gradient of $\phi_{1}[13,15]$,

$$
\min _{\phi_{1}(\mathbf{x})}: \mathcal{I}\left(\phi_{1}(\mathbf{x})\right)=\frac{1}{2} \int_{\Omega}\left\|\nabla \phi_{1}(\mathbf{x})-\mathbf{e}_{1}(\mathbf{x})\right\|^{2} \mathrm{~d} \Omega
$$

However, this is a best-fit that tries to take both the enforcement of the angle and lattice spacing into account simultaneously [16]. We will argue that the projected shape resembles the homogenizationbased topology best if the angles are enforced exactly, at the cost of a relaxed lattice spacing. To further enforce that the unit-cells are oriented corresponding to the output of the homogenization 
problem, $\phi_{1}$ has to be constant in the direction of $\mathbf{e}_{2}$. Equation 11 can thus be reformulated in the following constrained least-squares minimization problem.

$$
\begin{aligned}
\min _{\phi_{1}(\mathbf{x})}: & \mathcal{I}\left(\phi_{1}(\mathbf{x})\right)=\frac{1}{2} \int_{\Omega}\left\|\nabla \phi_{1}(\mathbf{x})-\mathbf{e}_{1}(\mathbf{x})\right\|^{2} \mathrm{~d} \Omega \\
\text { s.t. }: & \nabla \phi_{1}(\mathbf{x}) \cdot \mathbf{e}_{2}(\mathbf{x})=0
\end{aligned}
$$

Finally, it has to be mentioned that domain $\Omega$ can be split up into three parts. A void domain $\Omega_{v}$, a part of the domain that is completely solid $\Omega_{s}$, and a part that describes a lattice structure $\Omega_{l}$. These three different parts are defined as,

$$
\mathbf{x} \in\left\{\begin{array}{lll}
\Omega_{v} & \text { if } & m(\mathbf{x})=0 \\
\Omega_{s} & \text { if } & m(\mathbf{x})=1 \\
\Omega_{l} & \text { if } & 0<m(\mathbf{x})<1
\end{array}\right.
$$

The mapping $\phi_{1}$ should be just described accurately in $\Omega_{l}$, hence we can relax the accuracy of $\phi_{1}$ in $\Omega_{s}$ and $\Omega_{v}$ to make sure the best projection is obtained. To do this we reformulate the constrained least-squares minimization problem into a spatially weighted problem.

$$
\begin{aligned}
& \min _{\phi_{1}(\mathbf{x})}: \mathcal{I}\left(\phi_{1}(\mathbf{x})\right)=\frac{1}{2} \int_{\Omega} \alpha_{1}(\mathbf{x})\left\|\nabla \phi_{1}(\mathbf{x})-\mathbf{e}_{1}(\mathbf{x})\right\|^{2} \mathrm{~d} \Omega \\
& \text { s.t. : } \alpha_{2}(\mathbf{x}) \nabla \phi_{1}(\mathbf{x}) \cdot \mathbf{e}_{2}(\mathbf{x})=0
\end{aligned}
$$

where,

$$
\alpha_{1}(\mathbf{x})=\left\{\begin{array}{lll}
0.01 & \text { if } & \mathbf{x} \in \Omega_{v} \\
0.1 & \text { if } & \mathbf{x} \in \Omega_{s}, \\
1 & \text { if } & \mathbf{x} \in \Omega_{l}
\end{array} \quad \alpha_{2}(\mathbf{x})=\left\{\begin{array}{lll}
0 & \text { if } & \mathbf{x} \in \Omega_{v} \\
0 & \text { if } & \mathbf{x} \in \Omega_{s} \\
1 & \text { if } & \mathbf{x} \in \Omega_{l}
\end{array}\right.\right.
$$

The term $\alpha_{1}$ is introduced to relax the projection of $\phi_{1}$ in $\Omega_{v}$ and $\Omega_{s}$, where the low values still ensure some regularization to the lattice spacing. Furthermore, the term $\alpha_{2}$ is used to turn off exact angular enforcement in these regions. If equally weighted, requirements to the enforcement of angles in $\Omega_{v}$, where orientations may be badly determined, would severely influence the outcome in important higher density regions. Similarly, for $\Omega_{s}$, the orientation of the unit-cell is less important since it will neither affect the constitutive properties nor the projected shape. The Lagrangian corresponding to Equation 14 can thus be written as,

$$
\mathcal{L}(\phi 1(\mathbf{x}), \lambda(\mathbf{x}))=\int_{\Omega} \frac{\alpha_{1}(\mathbf{x})}{2}\left\|\nabla \phi_{1}(\mathbf{x})-\mathbf{e}_{1}(\mathbf{x})\right\|^{2}-\lambda(\mathbf{x}) \alpha_{2}(\mathbf{x})\left(\nabla \phi_{1}(\mathbf{x}) \cdot \mathbf{e}_{2}(\mathbf{x}) \mathrm{d} \Omega\right.
$$

where $\lambda$ is the Lagrange multiplier corresponding to the constraint.

\subsection{Projecting a rotationally symmetric spatially variant micro-structure}

The optimization problem solved in Equation 14 works well for a smoothly varying angle field $\theta$. Unfortunately, the principal stress directions used to calculate $\theta$ are rotationally symmetric, hence there may be jumps of size $\pi$ in the optimized angle field. This rotational symmetry has neither an influence on the shape of the micro-structure, nor on its constitutive properties. However, it means that Equation 12 cannot be used, unless vectors $\mathbf{e}_{1}$ and $\mathbf{e}_{2}$ are oriented in a consistent fashion. To 
circumvent this problem Pantz and Trabelsi [13] reformulated the problem and solved for vector fields $\mathbf{v}_{1}$ and $\mathbf{v}_{2}$ instead of the scalar fields $\phi_{1}$ and $\phi_{2}$.

We argue that the approach proposed by Pantz and Trabelsi overly complicates the procedure, and propose that the vector fields $\mathbf{e}_{1}$ and $\mathbf{e}_{2}$ can be oriented consistently in a straightforward fashion, in turn avoiding the computational burden in solving for vector fields $\mathbf{v}_{1}$ and $\mathbf{v}_{2}$. To do so we use a well-known image processing technique called connected component labeling [35]. The main idea is to divide the image into separate components, where the pixels in each of the components have uniform or near-uniform properties. Using this technique it is possible to find the components in the design domain where the angle field $\theta$ is rotated with $\pi$. To identify if voxel $i$ and adjacent voxel $j$ are in the same component, the following 2 conditions should be satisfied.

1. The relative difference between $\theta_{i}$ and $\theta_{j}$ should be smaller than $\pi / 2$.

2. Voxels $i$ and $j$ should both be in $\Omega_{v}$ or should both be in $\Omega_{s} \cup \Omega_{l}$

The first condition assumes that the principal stress field is smooth and should not rotate more than $\pi / 2$ between two adjacent elements. The second condition is used to find the boundaries of regions in which there is no material. This is important when considering the Michell-type problem with the circular support. Here we want the support to be a separate component that does not influence the orientation of angle field $\theta$ outside of the support. Connected component labeling is a well-known image processing task, for which various efficient implementations exist [35]. Furthermore, it has to be noted that the technique works equally well on irregular meshes, provided that the adjacent elements are known.

To demonstrate the procedure, consider the vector field $\mathbf{e}_{1}$ shown in Figure 6 (a). We assume a non-void domain, i.e. condition 2 is always satisfied, and perform the connected component label algorithm described above. A component label is assigned to each of the elements, and it can be seen in Figure 6 (b) that the edges of the components correspond to the places where angle field $\theta$ is rotated by $\pi$. The different components can then be oriented consistently by making sure that the difference in $\theta$ at these boundaries is close to $k 2 \pi$, where $k$ is an integer. This aligns the angle field in a consistent fashion, and hence the projection can be performed using Equation 12.

\subsection{Numerical implementation of the projection method}

The solution of the topology optimization problem, obtained on coarse mesh $\mathcal{T}^{c}$ is projected onto a fine scale mesh $\mathcal{T}^{f}$, where $h_{f}<h_{c} / 15$ to obtain a high-resolution design at a low computational cost. The calculation of $\phi_{1}$ and $\phi_{2}$ is done on an intermediate mesh $\mathcal{T}^{i}$, where the mesh-size is a trade-off between the computational cost and the quality of the result, however a general rule of thumb is that $h_{i}<h_{c} / 3[15]$. An overview of these three different meshes and how they are related can be found in Figure 7, where in the coarse mesh $\mathcal{T}^{c}$ the red lines correspond to $1-a_{1}$, while the blue lines correspond to $1-a_{2}$.

Different numerical methods exist to find $\phi_{1}$, and $\lambda$. Here we solve the minimization problem of Equation 14 in finite difference form, where the solution vectors $\phi_{1}$ and $\boldsymbol{\lambda}$, contain the corresponding unknowns on the intermediate mesh. The resulting discretized Lagrangian can be written as,

$$
\mathcal{L}\left(\phi_{1}, \boldsymbol{\lambda}\right)=\frac{1}{2}\left\|\mathbf{A}_{1}\left(\mathbf{D} \phi_{1}-\mathbf{E}\right)\right\|^{2}-\boldsymbol{\lambda}^{T} \mathbf{A}_{2} \mathbf{B D} \phi_{1}
$$

where $\mathbf{D}$ is a finite difference matrix, vector, $\mathbf{E}$ contains the unit vectors $\mathbf{e}_{1}$ at all discrete points, and matrix $\mathbf{B}$ holds the values of $\mathbf{e}_{2}$. It has to be noted that $\mathbf{A}_{1}$, and $\mathbf{A}_{2}$ are diagonal matrices such that the spatial weighting terms are taken into account, hence $\mathbf{A}_{1}$ contains values of $\sqrt{\alpha_{1}}$ at 


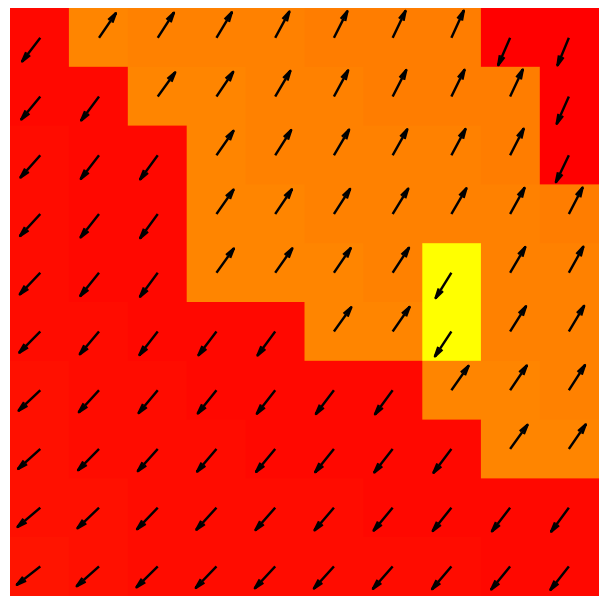

(a) $\mathbf{e}_{1}$ plotted on top of $\theta$

\begin{tabular}{|l|l|l|l|l|l|l|l|l|l|}
\hline 1 & 2 & 2 & 2 & 2 & 2 & 2 & 2 & 4 & 4 \\
\hline 1 & 1 & 2 & 2 & 2 & 2 & 2 & 2 & 2 & 4 \\
\hline 1 & 1 & 1 & 2 & 2 & 2 & 2 & 2 & 2 & 4 \\
\hline 1 & 1 & 1 & 2 & 2 & 2 & 2 & 2 & 2 & 2 \\
\hline 1 & 1 & 1 & 2 & 2 & 2 & 2 & 3 & 2 & 2 \\
\hline 1 & 1 & 1 & 1 & 1 & 2 & 2 & 3 & 2 & 2 \\
\hline 1 & 1 & 1 & 1 & 1 & 1 & 1 & 2 & 2 & 2 \\
\hline 1 & 1 & 1 & 1 & 1 & 1 & 1 & 1 & 2 & 2 \\
\hline 1 & 1 & 1 & 1 & 1 & 1 & 1 & 1 & 1 & 1 \\
\hline 1 & 1 & 1 & 1 & 1 & 1 & 1 & 1 & 1 & 1 \\
\hline
\end{tabular}

(b) Component label assigned to each element

Figure 6: Angle field $\theta$, which exhibits rotational symmetry, and the corresponding connected component labeling

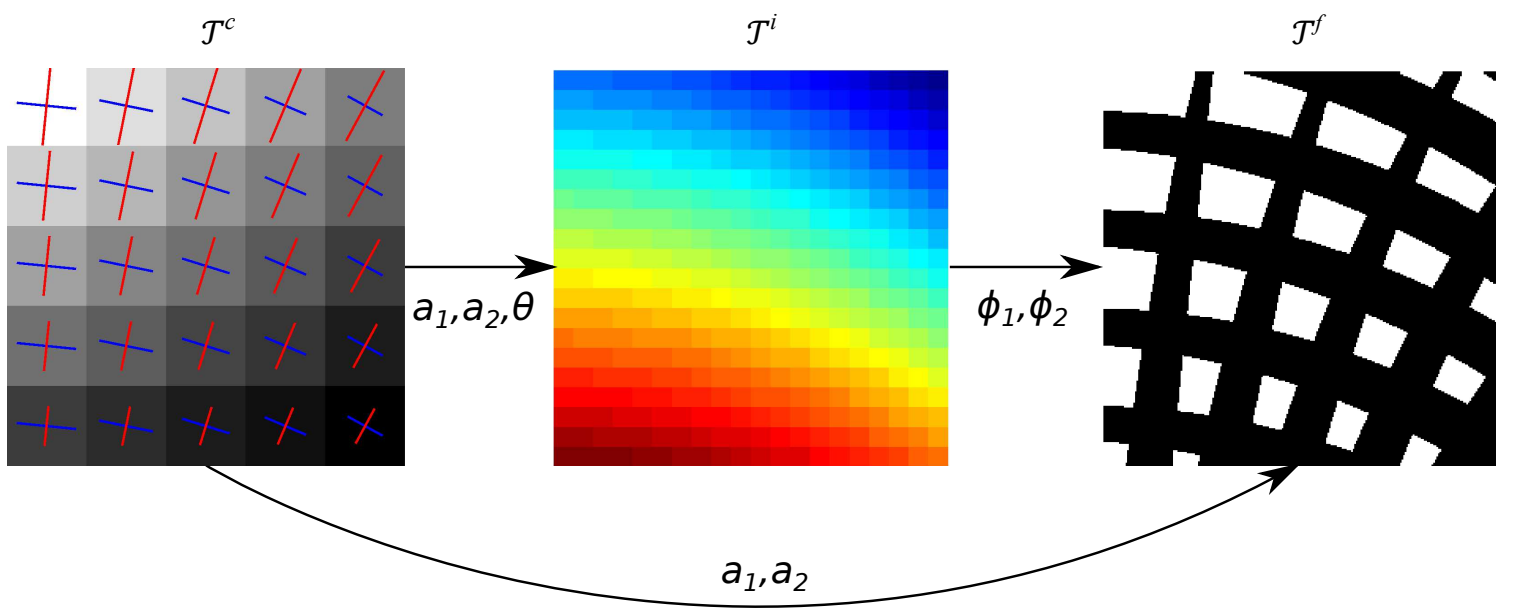

Figure 7: The different types of meshes and how they are linked.

the corresponding discrete points. Using the discretized Lagrangian and constraint, we can solve for $\phi_{1}$, and $\boldsymbol{\lambda}$ using the following KKT-system,

$$
\left[\begin{array}{cc}
\mathbf{D}^{T} \mathbf{A}_{1}^{T} \mathbf{A}_{1} \mathbf{D} & -\mathbf{D}^{T} \mathbf{B}^{T} \mathbf{A}_{2}^{T} \\
\mathbf{A}_{2} \mathbf{B D} & \mathbf{0}
\end{array}\right]\left[\begin{array}{c}
\phi_{1} \\
\boldsymbol{\lambda}
\end{array}\right]=\left[\begin{array}{c}
\mathbf{D}^{T} \mathbf{A}_{1}^{T} \mathbf{A}_{1} \mathbf{E} \\
\mathbf{0}
\end{array}\right]
$$

The solution of $\phi_{1}$ is prone to local high-frequency variations, as can be seen in Figure 8(a). These variations can be prevented by solving for $\phi_{1}$ on a finer mesh, however, this increases the cost of the projection. Furthermore, it was observed that smoothing $\phi_{1}$ resolved the issue equally well. To smooth scalar field $\phi_{1}$ a density filter with a radius of $2 h_{i}$ is used in a post-processing step. The 
effect of this smoothing operation can be seen in Figure 8(b).

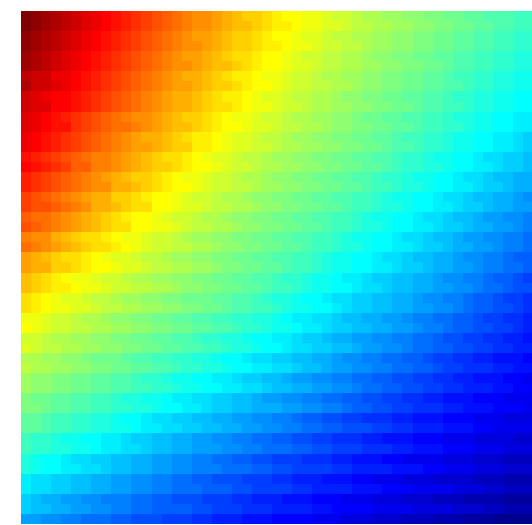

(a) $\phi_{1}$, with high-frequency variations

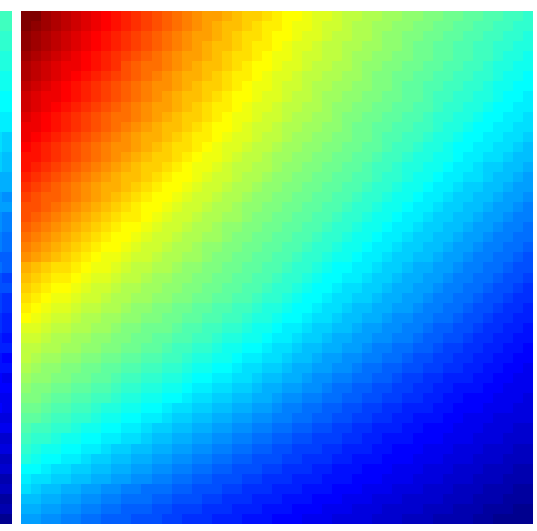

(b) $\phi_{1}$ after smoothing

Figure 8: Smoothing of field $\phi_{1}$ using a density filter.

\subsection{Scaling the periodicity}

The projection procedure, ensures that the local micro-structure is oriented with $\theta$, at the cost of the lattice spacing. The local lattice spacing is described by $\left\|\nabla \phi_{1}\right\|$ and $\left\|\nabla \phi_{2}\right\|$. If the angle is uniform throughout the domain, both values are 1 , since $\mathbf{e}_{1}$, and $\mathbf{e}_{2}$ are unit-vectors. Hence, $\left\|\nabla \phi_{i}\right\|>1$, means that cosine wave $i$ is locally compressed, while $\left\|\nabla \phi_{i}\right\|<1$, means that the cosine wave is locally stretched. To make sure that the average size of the projected unit-cell corresponds to $\varepsilon$, we have to find the average lattice spacing $\bar{p}_{i}$ in the part of the domain that consists of material $\left(\Omega \backslash \Omega_{v}\right)$.

$$
\bar{p}_{i}=\frac{\int_{\left(\Omega \backslash \Omega_{v}\right)}\left\|\nabla \phi_{i}(\mathbf{x})\right\| \mathrm{d}\left(\Omega \backslash \Omega_{v}\right)}{\int_{\left(\Omega \backslash \Omega_{v}\right)} \mathrm{d}\left(\Omega \backslash \Omega_{v}\right)}
$$

Hence, $\bar{p}_{i}>1$ means that cosine wave $i$ is compressed in the domain of interest. Therefore the periodicity for the $i$-th cosine wave $P_{i}$ has to be scaled using,

$$
P_{i}=\frac{2 \pi}{\varepsilon} \frac{1}{\bar{p}_{i}}
$$

The cantilever beam, for which the homogenization-based topology is shown in Figure 4, is treated as above. Here $\mathcal{T}^{c}$ consists of $80 \times 40$ elements, $\mathcal{T}^{i}$ consists of $320 \times 160$ elements, and the final shape shown in Figure 9(a) is obtained on a fine mesh consisting of $1600 \times 800$ elements, using $\varepsilon=15 h_{f}$. The projection resembles the optimized shape well, and the corresponding compliance calculated on the fine mesh $\mathcal{J}_{f}=63.6$. Similarly, the test problem has been projected for $\varepsilon=30 h_{f}$ as shown in Figure 9(b), with the corresponding compliance of 61.2. It is interesting to note that in this case a larger magnification leads to a better performing design. This is due to a larger number of small features being disconnected when $\varepsilon=15 h_{f}$. How to resolve these small and sometimes disconnected features is the subject of the next section. 


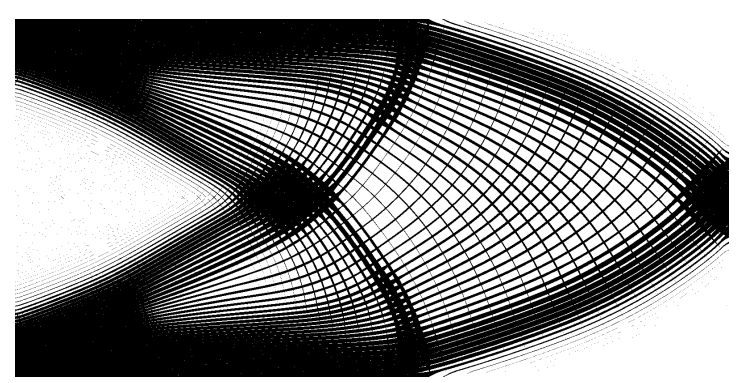

(a) $\varepsilon=15 h_{f}, \mathcal{J}_{f}=63.6$

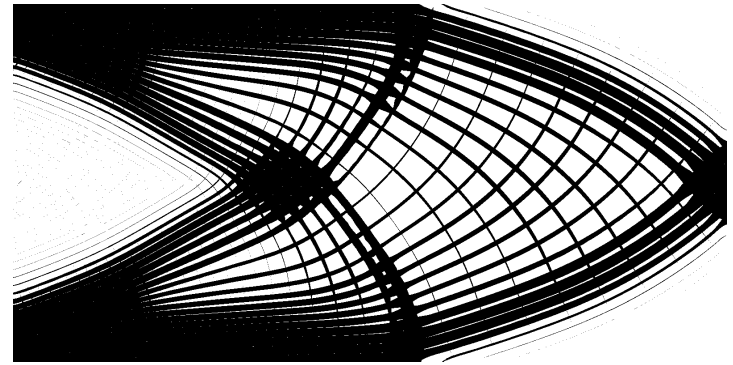

(b) $\varepsilon=30 h_{f}, \mathcal{J}_{f}=61.2$

Figure 9: Projection of the test problem on a fine scale of $1600 \times 800$ elements.

\section{Control of the shape of the projected micro-structure}

The size of the micro-structures can be varied using $\varepsilon$. However, this parameter does not provide any control of the feature size of the individual structural members. Furthermore, if we take a closer look at Figure 9(a) we can identify structural members that do not seem to carry any load, c.f. close-ups in Figure 10(a), as well as structural members that are so thin that they cease to exist, see Figure 10(b).

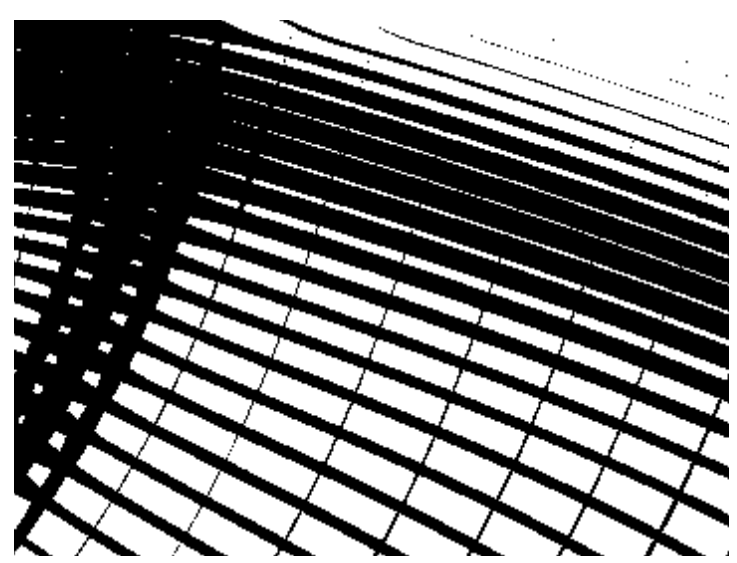

(a) Non-load carrying structural members

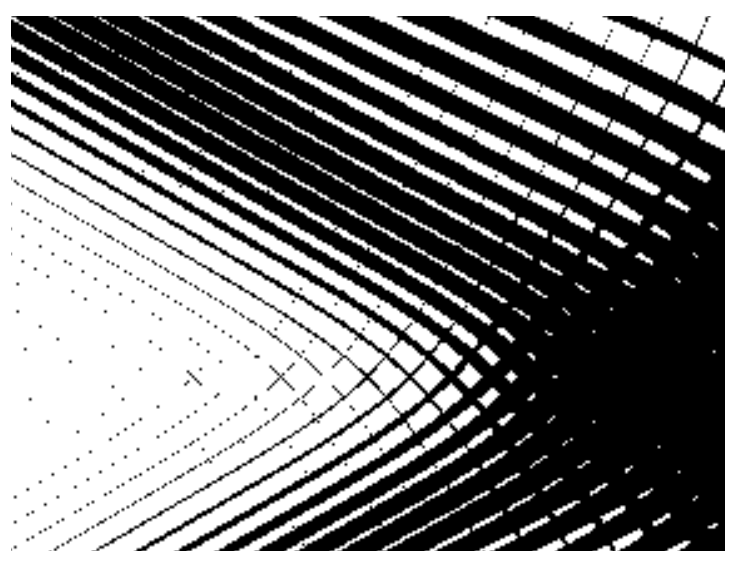

(b) Thin structural members

Figure 10: Close-up of the cantilever beam, projected on a fine scale of $1600 \times 800$ elements, with $\varepsilon=15 h_{f}$.

To avoid these undesired features, some control of the projected design is required. Therefore, we present a heuristic method that enforces a minimum feature size $f_{\text {min }}$ on both solid and void. First, we propose a continuation scheme that restricts the shape of the micro-structures to prevent the occurrence of unit-cells with very small structural members. Nevertheless, this approach still results in locations where the feature size of the projected design $f$, is smaller than $f_{\min }$, therefore material is added at these locations until manufacturability is ensured. Furthermore, we present a method which removes the material in non load-carrying regions. Finally, we show an overview of all steps that have to be taken to project the homogenization-based structure. 


\subsection{Restriction of solution space}

Micro-structures with value of $a_{1}$ and $a_{2}$ close but not equal to 0 or 1 have to be prevented. These values describe unit-cells with very thin strips of solid or void, which cannot be manufactured when the solution is projected on a fine scale. Instead we want these values to either be 0,1 or in a region $[\eta, 1-\eta]$, which is bounded by length-scale $\eta$ on both the solid and void. To get a solution that satisfies these conditions the following interpolation scheme is used, that links the filtered design variables $\tilde{a}_{1}$, and $\tilde{a}_{2}$ to the physical dimensions of the void $\overline{\tilde{a}}_{1}$, and $\overline{\tilde{a}}_{2}$.

$$
\overline{\tilde{a}}_{1}=\tilde{a}_{1}\left(1-H\left(\beta,(1-\eta), \tilde{a}_{1}\right)\right) H\left(\beta, \eta, \tilde{a}_{1}\right)+\left(\frac{\beta-1}{\beta}+\frac{\tilde{a}_{1}}{\beta}\right) H\left(\beta,(1-\eta), \tilde{a}_{1}\right)
$$

Where $H$ is the smoothed Heaviside function [36],

$$
H\left(\beta, \eta, \tilde{a}_{1}\right)=\frac{\tanh (\beta \eta)+\tanh \left(\beta\left(\tilde{a}_{1}-\eta\right)\right)}{\tanh (\beta \eta)+\tanh (\beta(1-\eta))}
$$

$\beta$ controls the sharpness of the projection, and $\eta$ controls the threshold value. For a high value of $\beta$ and $\eta=0$, we have that $\overline{\tilde{a}}_{1}=\tilde{a}_{1}$. The interpolation function for different values of $\beta$ and $\eta$ can be found in Figure 11. The order of lines in the legend shows the continuation approach that is taken, using 50 iterations per step. First, the material interpolation scheme is close to a linear function, but gradually $\eta$ is increased to enforce the length-scale on solid and void. Finally, $\beta$ is increased to ensure that the far majority of physical dimensions of the hole are either 0,1 or in the region $[\eta, 1-\eta]$, such that the small feature sizes are avoided.
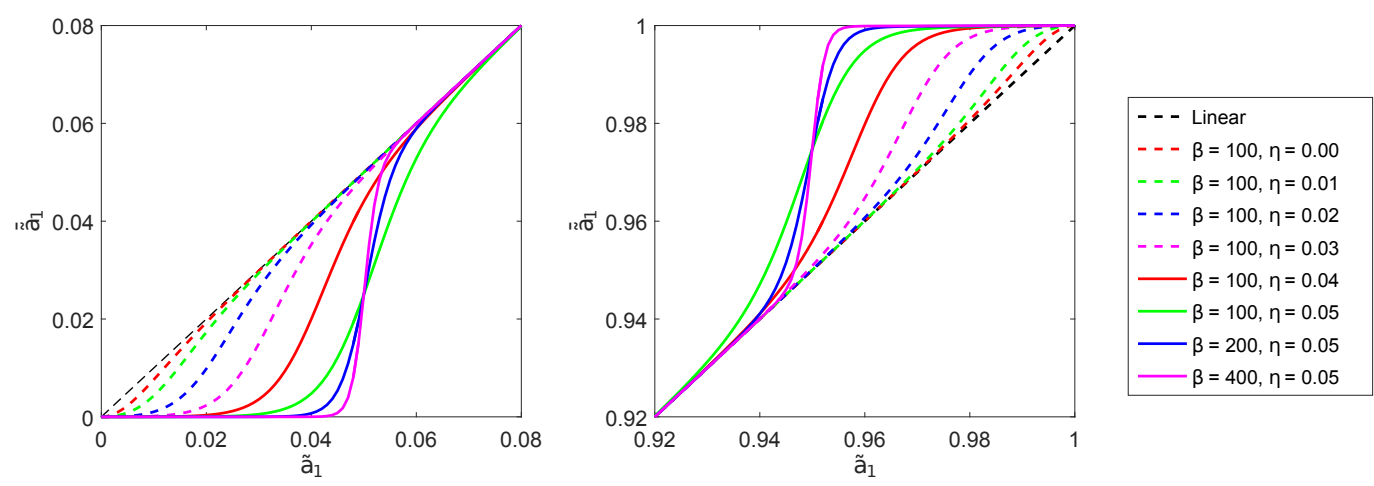

Figure 11: Interpolation scheme plotted for the intervals where the behavior is non-linear, for different values of $\eta$ and $\beta$, that follow the order of the continuation approach.

The cantilever beam problem has been solved using the continuation approach resulting in a compliance of 58.31 compared to the compliance of 58.35 using only density filtering. The difference between these values is negligible, similar differences have been observed for different mesh-sizes and different optimization problems. This shows that even though the solution space is restricted, equally well performing topologies can be found at different local minima. Hence, based on our experience, the presented approach does not negatively affect the performance of the design for the chosen parameters. 


\subsection{Satisfying the local feature-sizes}

The presented restriction method greatly reduces the violation of length-scale $f_{\min }$ in the projected design. Nevertheless, it does not enforce an explicit length-scale on the projected shape. It is possible to identify the projected feature-sizes $f_{s}$, and $f_{v}$, corresponding to the solid and void respectively, using the local periodicity and $a_{1}$, and $a_{2}$.

$$
\begin{gathered}
f_{s, i}(\mathbf{x})=\frac{\varepsilon\left(1-a_{i}(\mathbf{x})\right)}{\left\|\nabla \phi_{i}(\mathbf{x})\right\|} \\
f_{v, i}(\mathbf{x})=\frac{\varepsilon a_{i}(\mathbf{x})}{\left\|\nabla \phi_{i}(\mathbf{x})\right\|}
\end{gathered}
$$

If $f_{s, i}(\mathbf{x})$ is smaller than $f_{\min }$, the feature size of the solid is violated. To compensate for this, a new value $a_{i}^{*}$ is found such that the length-scale is satisfied.

$$
a_{i}^{*}(\mathbf{x})=1-\frac{f_{\min }}{\varepsilon}\left\|\nabla \phi_{i}(\mathbf{x})\right\|
$$

Similarly, if $f_{v, i}<f_{\min }$, the hole is too small. However, we observed that removing material to satisfy the length-scale has a significant negative effect on the optimality of the projected shape. Therefore, to make sure that the feature size is satisfied, the hole is closed instead, i.e. $a_{i}^{*}=0$. This approach adds material to the structure and thus violates the volume constraint $V_{\max }$ used in the optimization problem. Nevertheless, we argue that this violation is small, e.g. $5 \%$ of $V_{\max }$, if reasonable values for $\varepsilon$, and $f_{\min }$ are used. The restriction of the solution space discussed previously, makes sure that most of the unit-cells in the domain do not have small feature sizes. Furthermore, the non-load carrying structural members shown in Figure 10(a) still have to be removed, which will reduce the violation of the volume constraint.

\subsection{Removal of material at non-load carrying solids}

Non-load carrying structural members, see e.g. Figure 10(a), can partially be explained by using the restriction method presented above, but mostly by the interpolation of $a_{1}$ and $a_{2}$ from $\mathcal{T}^{c}$ onto $\mathcal{T}^{f}$. Since a linear interpolation method is used, isolated structural members can arise at high-contrast regions. To remove these structural members we use a simple iterative update scheme. In this scheme a finite element analysis is performed at the fine-scale, afterwards the solid voxels that have a strain energy density $c_{e}$ lower than $0.1 \%$ of the mean strain energy density $\overline{c_{e}}$ are set to void. To make sure that the length-scale $f_{\min }$ on both the solid and void is still satisfied after each iteration, we perform an open-close filter operation c.f. [37]. This operation efficiently eliminates features smaller than the filter size but leaves other details virtually untouched.

The combination of removal of solid voxels with a low strain energy density, followed by the openclose filter operation, is a heuristic method to get smooth and manufacturable micro-structures. Nevertheless, we have observed excellent behavior of this method and generally convergence is obtained within 5-10 iterations. Alternatively, one could use the projected micro-structure as a starting guess for fine-scale topology optimization. However, even when an efficient topology optimization framework is used, c.f. [38], the corresponding optimization will require significantly more time consuming fine-scale iterations until convergence, and probably result in no significant improvement in performance. 


\subsection{Overview of the projection procedure}

The methods described in the previous sections are combined to obtain high-resolution manufacturable micro-structures at low computational cost. The different steps that have to be taken are shown below:

1. Homogenization-based topology optimization for rectangular unit-cells using the restriction approach presented in Section 4.1, on coarse mesh $\mathcal{T}^{c}$.

2. Connected component labeling and consistent alignment of $\mathbf{e}_{1}$ and $\mathbf{e}_{2}$, using the methodology presented in Section 3.3 on $\mathcal{T}^{c}$.

3. Calculating mapping functions $\phi_{1}$, and $\phi_{2}$ as shown in Section 3.4, on intermediate mesh $\mathcal{T}^{i}$.

4. Satisfying feature-size $f$, using the method presented in Section 4.2, on fine mesh $\mathcal{T}^{f}$.

5. Projection of the cosine waves using Equations 7- 10 on fine mesh $\mathcal{T}^{f}$.

6. Removal of the non-load carrying materials using the iterative method described in Section 4.3 on mesh $\mathcal{T}^{f}$.

\section{Numerical examples}

The solution of the cantilever beam problem has been projected on a fine mesh consisting of $1600 \times 800$ elements. The homogenization-based topology optimization took $199.4 \mathrm{~s}$, and the subsequent connected component labeling $0.1 \mathrm{~s}$. Hence, the total time spent on the coarse mesh $T_{c}=199.5 \mathrm{~s}$. The compliance of the optimized homogenization-based topology $\mathcal{J}_{c}=58.31$. The projection is performed for various sizes of the unit-cell $\varepsilon$, and several values of $f_{\text {min }}$. The corresponding compliance on the fine-scale $\mathcal{J}_{f}$, and volume $V_{f}$ of the projected structures can be found in Table 1. Furthermore, $T_{i}$ which is the time it took to calculate $\phi_{1}$ and $\phi_{2}$ on the intermediate mesh is shown, as well as $T_{f}$ the time it took to satisfy the feature size, project the cosine waves, and remove the non-load carrying members on the fine mesh. Finally, the time for the entire procedure $T_{t o t}$ is given in Table 1.

Table 1: Performance and computational cost of the projection method, when the cantilever beam problem is projected on a fine mesh consisting of $1600 \times 800$ elements.

\begin{tabular}{ccccccccc}
\hline$\varepsilon$ & $f_{\min }$ & $V_{f}$ & $\mathcal{J}_{c}$ & $\mathcal{J}_{f}$ & $T_{c}$ & $T_{i}$ & $T_{f}$ & $T_{\text {tot }}$ \\
\hline $20 h_{f}$ & $2 h_{f}$ & 0.503 & 58.31 & 60.27 & $199.5 \mathrm{~s}$ & $10.7 \mathrm{~s}$ & $145.2 \mathrm{~s}$ & $355.4 \mathrm{~s}$ \\
$20 h_{f}$ & $3 h_{f}$ & 0.519 & 58.31 & 58.21 & $199.5 \mathrm{~s}$ & $10.2 \mathrm{~s}$ & $260.6 \mathrm{~s}$ & $470.3 \mathrm{~s}$ \\
$20 h_{f}$ & $4 h_{f}$ & 0.540 & 58.31 & 56.23 & $199.5 \mathrm{~s}$ & $10.8 \mathrm{~s}$ & $266.6 \mathrm{~s}$ & $476.9 \mathrm{~s}$ \\
$30 h_{f}$ & $2 h_{f}$ & 0.500 & 58.31 & 60.93 & $199.5 \mathrm{~s}$ & $10.7 \mathrm{~s}$ & $150.3 \mathrm{~s}$ & $360.5 \mathrm{~s}$ \\
$30 h_{f}$ & $3 h_{f}$ & 0.509 & 58.31 & 59.72 & $199.5 \mathrm{~s}$ & $11.2 \mathrm{~s}$ & $233.6 \mathrm{~s}$ & $444.3 \mathrm{~s}$ \\
$30 h_{f}$ & $4 h_{f}$ & 0.518 & 58.31 & 58.71 & $199.5 \mathrm{~s}$ & $10.5 \mathrm{~s}$ & $285.3 \mathrm{~s}$ & $495.3 \mathrm{~s}$ \\
$40 h_{f}$ & $2 h_{f}$ & 0.500 & 58.31 & 59.55 & $199.5 \mathrm{~s}$ & $10.7 \mathrm{~s}$ & $115.5 \mathrm{~s}$ & $325.7 \mathrm{~s}$ \\
$40 h_{f}$ & $3 h_{f}$ & 0.505 & 58.31 & 59.03 & $199.5 \mathrm{~s}$ & $10.2 \mathrm{~s}$ & $145.6 \mathrm{~s}$ & $355.3 \mathrm{~s}$ \\
$40 h_{f}$ & $4 h_{f}$ & 0.510 & 58.31 & 58.57 & $199.5 \mathrm{~s}$ & $10.0 \mathrm{~s}$ & $148.7 \mathrm{~s}$ & $358.2 \mathrm{~s}$ \\
\hline
\end{tabular}

A small unit-cell size combined with a large minimum length-scale, leads to a large violation of the volume constraint. This is visualized for $\varepsilon=20 h_{f}$ and $f_{\min }=4 h_{f}$ in Figure 12(a) , where most structural members have a width corresponding to $f_{\text {min }}$. However, for reasonable combinations of $\varepsilon$, and $f_{\text {min }}$ the volume constraint is hardly violated. A good rule of thumb is that 
$\frac{f_{\min }}{\varepsilon}>10$ such that the violation of the volume constraint is within $2 \%$ of $V_{\max }$. Furthermore, it is very interesting to see that for these projected structures excellent results are achieved, where the compliance is close to the performance of the homogenization-based topology. For $\varepsilon=40$, and a small feature-size $f_{\min }=2$, we obtain a structure that does not violate the volume constraint, see Figure 12(b), while the performance is within $2 \%$ of the homogenization-based topology. Using a larger feature-size $f_{\text {min }}=4$, slightly increases the volume, however, this results in a topology for which the performance is almost identical to the homogenization-based topology. Furthermore, this larger feature-size ensures manufacturability as can be seen in Figure 13.

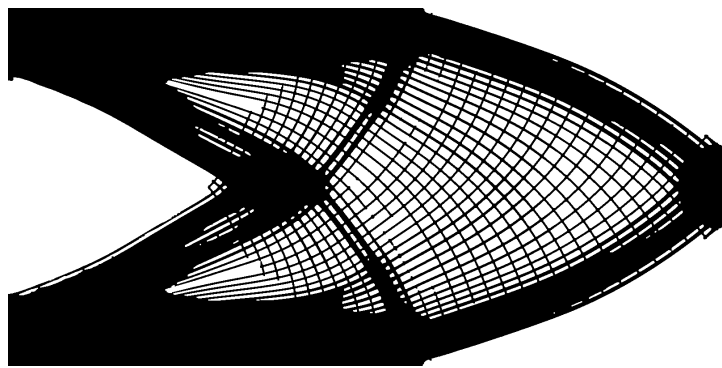

(a) $\varepsilon=20 h_{f}, f_{\text {min }}=4 h_{f}, \mathcal{J}_{f}=56.23$, and $V_{f}=$ 0.540

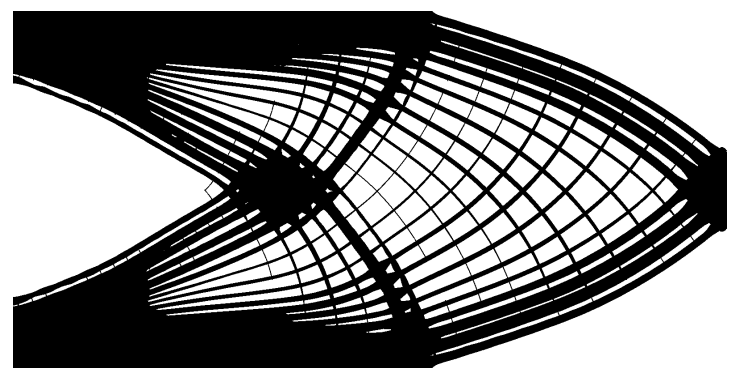

(b) $\varepsilon=40 h_{f}, f_{\text {min }}=2 h_{f}, \mathcal{J}_{f}=59.55$, and $V_{f}=$ 0.500 .

Figure 12: Cantilever problem projected on a fine scale of $1600 \times 800$

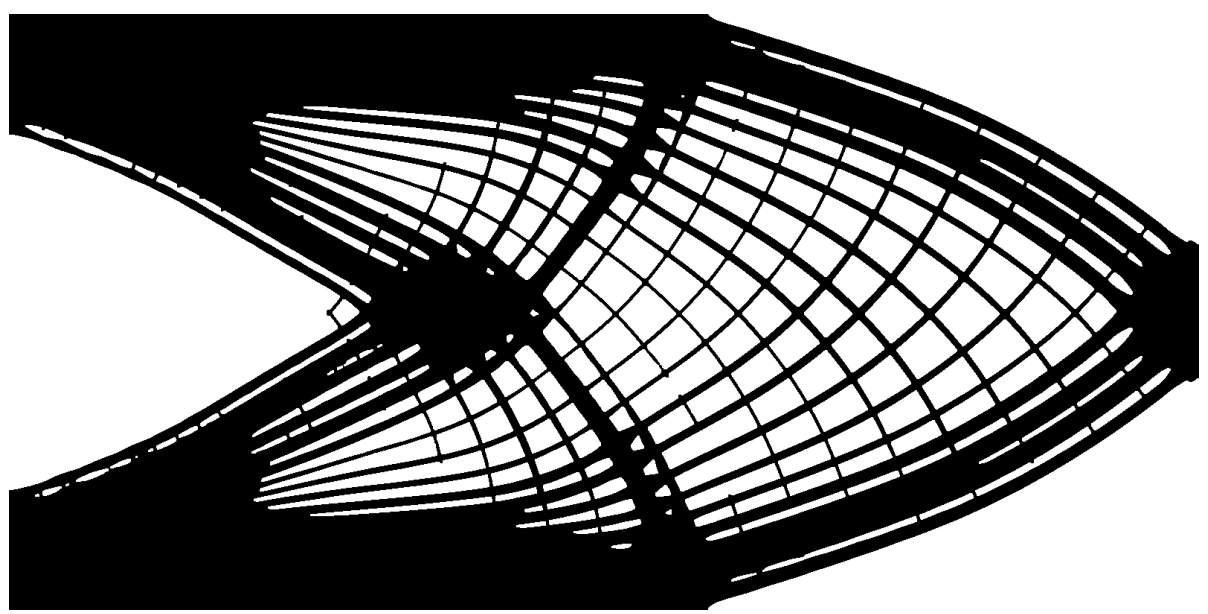

Figure 13: Projection of the cantilever problem, on a fine scale of $1600 \times 800$ using $\varepsilon=40 h_{f}$, and $f_{\text {min }}=4 h_{f}$. The compliance $\mathcal{J}_{f}=58.57$, while $V_{f}=0.510$

The presented procedure for calculating mapping functions $\phi_{1}$ and $\phi_{2}$ is highly efficient resulting in an average time of $10.6 \mathrm{~s}$. The time it took to project the structure on the fine-scale and satisfy the feature size is negligible compared to the cost of removing the non-load carrying materials. The cost for this iterative procedure depends on the required number of iterations, where an average iteration costs approximately $28 \mathrm{~s}$. This means that the total time to optimize and project the design 
$T_{\text {tot }}$ never exceeded $500 \mathrm{~s}$, where it has to be noted that all experiments have been performed on a standard PC, using a single-core MATLAB code.

To require the same resolution using a standard density-based topology optimization approach, a minimum of 100 iterations is required, each costing approximately 25 seconds. Furthermore, for a standard topology optimization approach a continuation strategy is required to get as close to the optimal solution as obtained by the suggested projection approach. Such a continuation approach requires approximately 1000 iterations [34], which would mean a total optimization time of approximately 25000 seconds. Due to its optimization at a coarse-scale the presented method is thus able to be reduce the computational cost by more than 50 times!

The proposed scheme is also applied to the Michell-type problem with circular support. The coarse-scale optimization performed on a mesh of $80 \times 60$ elements resulted in a compliance of 84.31 obtained within $225.4 \mathrm{~s}$. The shape is projected on a fine mesh of $1200 \times 900$ elements, for various sizes of the unit-cell $\varepsilon$, and several values of $f_{\min }$. The corresponding volume, compliance and times it took for each part of the procedure are shown in Table 2

Table 2: Performance and computational cost of the projection method, when the Michell-type problem with circular support is projected on a fine mesh consisting of $1200 \times 900$ elements.

\begin{tabular}{ccccccccc}
\hline$\varepsilon$ & $f_{\min }$ & $V_{f}$ & $\mathcal{J}_{c}$ & $\mathcal{J}_{f}$ & $T_{c}$ & $T_{i}$ & $T_{f}$ & $T_{\text {tot }}$ \\
\hline $20 h_{f}$ & $2 h_{f}$ & 0.248 & 84.31 & 75.57 & $225.6 \mathrm{~s}$ & $16.8 \mathrm{~s}$ & $109.6 \mathrm{~s}$ & $352.0 \mathrm{~s}$ \\
$20 h_{f}$ & $3 h_{f}$ & 0.266 & 84.31 & 73.44 & $225.6 \mathrm{~s}$ & $17.5 \mathrm{~s}$ & $112.7 \mathrm{~s}$ & $355.8 \mathrm{~s}$ \\
$20 h_{f}$ & $4 h_{f}$ & 0.297 & 84.31 & 62.76 & $225.6 \mathrm{~s}$ & $16.1 \mathrm{~s}$ & $150.4 \mathrm{~s}$ & $392.1 \mathrm{~s}$ \\
$30 h_{f}$ & $2 h_{f}$ & 0.236 & 84.31 & 76.68 & $225.6 \mathrm{~s}$ & $17.7 \mathrm{~s}$ & $174.2 \mathrm{~s}$ & $417.5 \mathrm{~s}$ \\
$30 h_{f}$ & $3 h_{f}$ & 0.241 & 84.31 & 74.72 & $225.6 \mathrm{~s}$ & $17.5 \mathrm{~s}$ & $132.5 \mathrm{~s}$ & $375.6 \mathrm{~s}$ \\
$30 h_{f}$ & $4 h_{f}$ & 0.252 & 84.31 & 71.89 & $225.6 \mathrm{~s}$ & $15.7 \mathrm{~s}$ & $175.3 \mathrm{~s}$ & $416.6 \mathrm{~s}$ \\
$40 h_{f}$ & $2 h_{f}$ & 0.250 & 84.31 & 68.37 & $225.6 \mathrm{~s}$ & $16.5 \mathrm{~s}$ & $111.6 \mathrm{~s}$ & $353.7 \mathrm{~s}$ \\
$40 h_{f}$ & $3 h_{f}$ & 0.252 & 84.31 & 67.83 & $225.6 \mathrm{~s}$ & $17.3 \mathrm{~s}$ & $112.6 \mathrm{~s}$ & $355.5 \mathrm{~s}$ \\
$40 h_{f}$ & $4 h_{f}$ & 0.256 & 84.31 & 66.51 & $225.6 \mathrm{~s}$ & $17.7 \mathrm{~s}$ & $92.9 \mathrm{~s}$ & $336.2 \mathrm{~s}$ \\
\hline
\end{tabular}

It is interesting to see that for $\varepsilon=30 h_{f}$ the volume of the projected and post-processed shape is smaller than the volume constraint in the topology optimization problem. The reason for this is that two peaks of the cosine waves are located exactly at the boundary of the void domain and are not well connected to the rest of the structure, as can be seen in Figure 14(a). Therefore these waves are non-load carrying and removed by the fine-scale optimization procedure as can be seen in Figure 14(b). This unfortunate result is purely a problem of the periodicity $P$ having an unlucky value such that the projected structure at some positions is not well connected. If we use $\varepsilon=40 h_{f}$ the peaks of the cosine waves are positioned in a better way and excellent results are obtained. The projection using $40 h_{f}$ and $3 h_{f}$ is shown in Figure 15, the violation of the volume constraint is negligible and the corresponding compliance is 67.83 . It is interesting that this value is much lower than the compliance of the homogenization based topology. This difference can be explained by the enforcement of the circular boundary condition, which is known to have a very strong influence on the resulting compliance for this classical example. If the homogenization-based topology optimization is performed on a finer scale the boundary is approximated more accurately, and it was observed that the compliance converges to the value obtained using the projection.

From the numerical examples we can conclude that the presented method is able to provide high-resolution (more than a million elements), near-optimal, manufacturable topologies, at low computational cost. However, the exact locations of the peaks of the cosine-waves are difficult to 


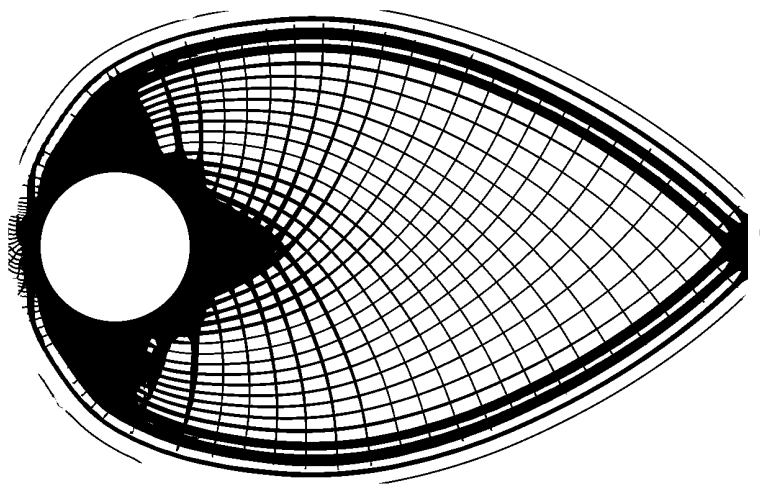

(a) Before removal of non-load carrying material

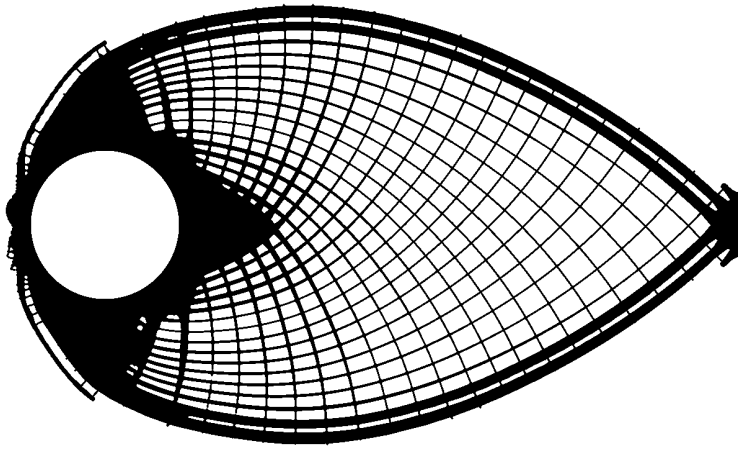

(b) After removal of non-load carrying material

Figure 14: The Michell-type problem with circular support projected on a fine mesh consisting of $1200 \times 900$ elements. $\varepsilon=30 h_{f}$, and $f_{\min }=3 h_{f}$.

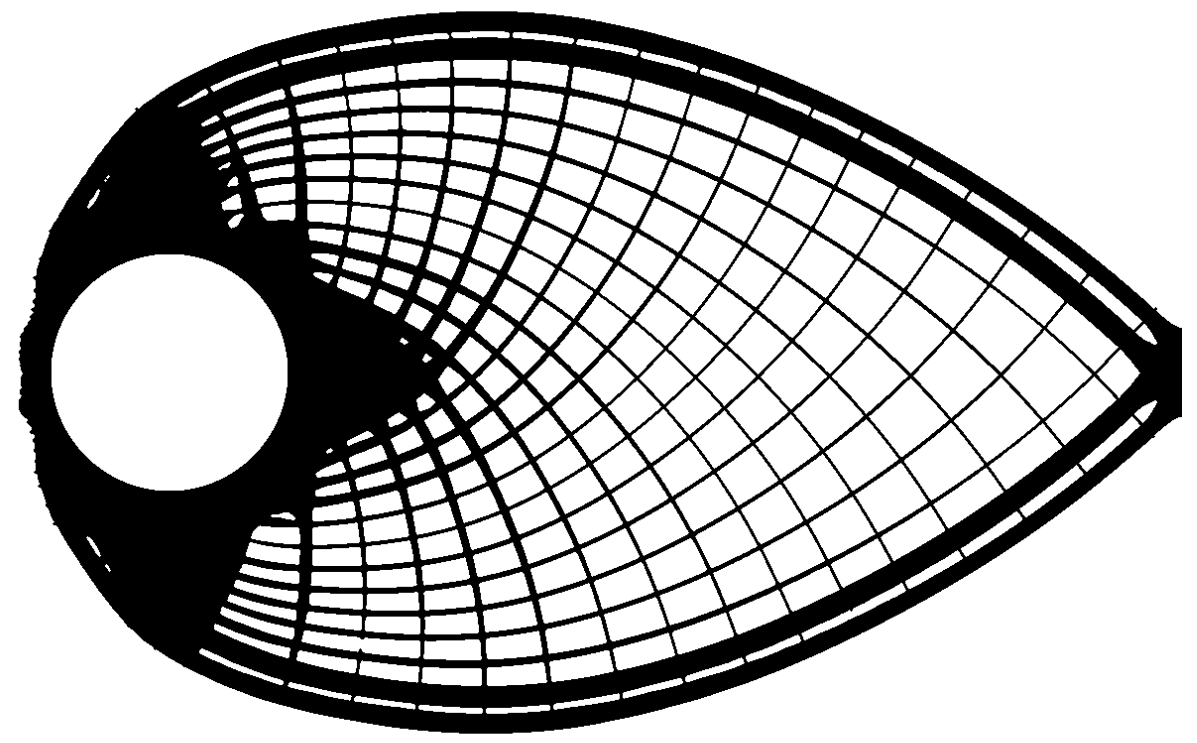

Figure 15: The Michell-type problem with circular support projected on a fine mesh consisting of $1200 \times 900$ elements. $\varepsilon=40 h_{f}$, and $f_{\min }=3 h_{f}$, the compliance $\mathcal{J}_{f}=67.83$, while $V_{f}=0.252$

predict, therefore the projected shape can have some poorly connected structural members, as is demonstrated in Figure 14(a) and (b). This problem can be circumvented by shifting the cosine waves slightly, or by solving the projection procedure as a true multi-scale approach. In such an approach the topology optimized shape, is also the shape that can be projected best. 
Finally, a close inspection of Figures 13 and 15 shows that the projected shapes are not perfectly symmetric. The reason for this are angular fluctuations in low density regions. The influence of these regions on the projection is small, nevertheless, it can still result in a slight asymmetry as is best visible around the circular support in Figure 15. This asymmetry can be circumvented by reducing the computational domain at which the projection is performed to the non-void regions or by enforcing symmetry in all variable sets. We will leave further improvements in this direction to future works.

\section{Conclusion}

An efficient approach to obtain near-optimal, high-resolution, manufacturable micro-structures from coarse scale optimization studies has been presented. The methodology projects the unitcells of homogenization-based topology optimization on a fine mesh with minimum computational effort, where emphasis has been put on how to treat the rotational symmetry in the optimized angle field. Furthermore, an efficient method to control the shape of the projected design has been presented, such that the final topology satisfies a length-scale on both solid and void regions. Based on numerical experiments, this heuristic method has shown to produce near-optimal designs, at a speed which for the considered 2D examples is 50 times lower than conventional density-based topology optimization methods.

This overall promising performance paves the way for extending the proposed methodology to multi-load problems, where more complex unit-cells need to be considered. Furthermore, the excellent performance for single load-case minimum compliance problems, allows for a natural extension of the methodology to 3D applications, where cubic unit-cells with rectangular holes will have to be considered. We are confident that the presented methodology is robust enough to allow these extensions, such that its potential for incorporation in an efficient, interactive design process can be further revealed.

\section{Acknowledgement}

The authors acknowledge the support of the Villum Fonden through the NextTop project. The authors would also like to thank Jens Gravesen, and Boyan Lazarov for valuable discussions during the preparation of the work. Finally, the authors wish to thank Krister Svanberg for the MATLAB MMA code. 
[1] N. Aage, E. Andreassen, B. Lazarov, Topology optimization using petsc: An easy-to-use, fully parallel, open source topology optimization framework, Structural and Multidisciplinary Optimization 51 (3) (2015) 565-572. doi:10.1007/s00158-014-1157-0.

[2] M. Bendsøe, N. Kikuchi, Generating optimal topologies in structural design using a homogenization method, Computer Methods in Applied Mechanics and Engineering 71 (2) (1988) 197-224. doi : 10.1016/0045-7825 (88)90086-2.

[3] H. Rodrigues, J. Guedes, M. Bendsøe, Hierarchical optimization of material and structure, Structural and Multidisciplinary Optimization 24 (1) (2002) 1-10. doi : 10.1007/s00158-002-0209-z.

[4] P. Coelho, J. Cardoso, P. Fernandes, H. Rodrigues, Parallel computing techniques applied to the simultaneous design of structure and material, Advances in Engineering Software $42(5)$ (2011) 219-227. doi:10.1016/j.advengsoft.2010.10.003.

[5] L. Xia, P. Breitkopf, Recent advances on topology optimization of multiscale nonlinear structures, Archives of Computational Methods in Engineering (2016) 123doi: $10.1007 / \mathrm{s} 11831-016-9170-7$.

[6] F. Schury, M. Stingl, F. Wein, Efficient two-scale optimization of manufacturable graded structures, SIAM Journal on Scientific Computing 34 (6) (2012) B711-B733. doi: $10.1137 / 110850335$.

[7] R. Sivapuram, P. Dunning, H. Kim, Simultaneous material and structural optimization by multiscale topology optimization, Structural and Multidisciplinary Optimization (2016) 115doi:10.1007/s00158-016-1519-x.

[8] J. Greifenstein, M. Stingl, Simultaneous parametric material and topology optimization with constrained material grading, Structural and Multidisciplinary Optimization (2016) 114doi: $10.1007 / \mathrm{s} 00158-016-1457-7$.

[9] A. Clausen, N. Aage, O. Sigmund, Topology optimization of coated structures and material interface problems, Computer Methods in Applied Mechanics and Engineering 290 (2015) 524541. doi:10.1016/j.cma.2015.02.011.

[10] A. Clausen, N. Aage, O. Sigmund, Exploiting additive manufacturing infill in topology optimization for improved buckling load, Engineering 2 (2016) 250-257. doi:10.1016/J.ENG.2016.02.006.

[11] B. Lazarov, Topology Optimization Using Multiscale Finite Element Method for HighContrast Media, Springer Berlin Heidelberg, Berlin, Heidelberg, 2014, pp. 339-346. doi : 10.1007/978-3-662-43880-0_38.

[12] J. Alexandersen, B. Lazarov, Topology optimisation of manufacturable microstructural details without length scale separation using a spectral coarse basis preconditioner, Computer Methods in Applied Mechanics and Engineering 290 (2015) 156 - 182. doi:10.1016/j.cma.2015.02.028. 
[13] O. Pantz, K. Trabelsi, A post-treatment of the homogenization method for shape optimization, SIAM Journal on Control and Optimization 47 (3) (2008) 1380-1398. doi:10.1137/070688900.

[14] O. Pantz, K. Trabelsi, Construction of minimization sequences for shape optimization, in: Methods and Models in Automation and Robotics (MMAR), 2010 15th International Conference on, 2010, pp. 278-283. doi:10.1109/MMAR.2010.5587222.

[15] R. Rumpf, J. Pazos, Synthesis of spatially variant lattices, Optics Express 20 (14) (2012) 15263-15274. doi:10.1364/OE.20.015263.

[16] R. Rumpf, J. Pazos, J. Digaum, S. Kuebler, Spatially variant periodic structures in electromagnetics, Philosophical Transaction Of The Royal Society A 373 (2049) (2015) 1-22. doi:10.1098/rsta.2014.0359.

[17] N. Aage, M. Nobel-Jørgensen, C. S. Andreasen, O. Sigmund, Interactive topology optimization on hand-held devices, Structural and Multidisciplinary Optimization 47 (1) (2013) 1-6. doi : 10.1007/s00158-012-0827-z.

[18] K.-T. Cheng, N. Olhoff, An investigation concerning optimal design of solid elastic plates, International Journal of Solids and Structures 17 (3) (1981) 305 - 323. doi: 10.1016/0020-7683(81)90065-2.

[19] G. A. Francfort, F. Murat, Homogenization and optimal bounds in linear elasticity, Archive for Rational Mechanics and Analysis 94 (4) (1986) 307-334. doi:10.1007/BF00280908.

[20] M. Avellaneda, Optimal bounds and microgeometries for elastic two-phase composites, SIAM Journal on Applied Mathematics 47 (6) (1987) 1216-1228. doi:10.1137/0147082.

[21] M. P. Bendsøe, Optimal shape design as a material distribution problem, Structural optimization 1 (4) (1989) 193-202. doi:10.1007/BF01650949.

[22] J. Guedes, N. Kikuchi, Preprocessing and postprocessing for materials based on the homogenization method with adaptive finite element methods, Computer Methods in Applied Mechanics and Engineering 83 (2) (1990) 143 - 198. doi:10.1016/0045-7825(90)90148-F.

[23] B. Hassani, E. Hinton, A review of homogenization and topology optimization I homogenization theory for media with periodic structure, Computers \& Structures 69 (6) (1998) 707 - 717. doi : $10.1016 /$ S0045-7949(98)00131-X.

[24] B. Hassani, E. Hinton, A review of homogenization and topology optimization II analytical and numerical solution of homogenization equations, Computers \& Structures 69 (6) (1998) 719 - 738. doi :10.1016/S0045-7949(98)00132-1.

[25] B. Hassani, E. Hinton, A review of homogenization and topology optimization III topology optimization using optimality criteria, Computers \& Structures 69 (6) (1998) 739 - 756 . doi : $10.1016 /$ S0045-7949(98) 00133-3.

[26] E. Andreassen, C. Andreasen, How to determine composite material properties using numerical homogenization, Computational Materials Science 83 (2014) 488 - 495. doi:10.1016/j.commatsci.2013.09.006. 
[27] O. Sigmund, K. Maute, Topology optimization approaches, Structural and Multidisciplinary Optimization 48 (6) (2013) 1031-1055. doi:10.1007/s00158-013-0978-6.

[28] P. Pedersen, On optimal orientation of orthotropic materials, Structural optimization 1 (2) (1989) 101-106. doi:10.1007/BF01637666.

[29] P. Pedersen, Bounds on elastic energy in solids of orthotropic materials, Structural optimization 2 (1) (1990) 55-63. doi:10.1007/BF01743521.

[30] K. Svanberg, The method of moving asymptotesa new method for structural optimization, International Journal for Numerical Methods in Engineering 24 (2) (1987) 359-373. doi:10.1002/nme.1620240207.

[31] A. Díaz, O. Sigmund, Checkerboard patterns in layout optimization, Structural optimization 10 (1) (1995) 40-45. doi:10.1007/BF01743693.

[32] B. Bourdin, Filters in topology optimization, International Journal for Numerical Methods in Engineering 50 (9) (2001) 2143-2158. doi:10.1002/nme.116.

[33] T. Bruns, D. Tortorelli, Topology optimization of non-linear elastic structures and compliant mechanisms, Computer Methods in Applied Mechanics and Engineering 190 (2627) (2001) 3443 - 3459. doi:10.1016/S0045-7825(00) 00278-4.

[34] O. Sigmund, N. Aage, E. Andreassen, On the (non-)optimality of michell structures, Structural and Multidisciplinary Optimization 54 (2) (2016) 361-373. doi:10.1007/s00158-016-1420-7.

[35] L. G. Shapiro, G. Stockman, Computer Vision, 1st Edition, Prentice Hall PTR, Upper Saddle River, NJ, USA, 2001.

[36] F. Wang, B. Lazarov, O. Sigmund, On projection methods, convergence and robust formulations in topology optimization, Structural and Multidisciplinary Optimization 43 (6) (2011) 767-784. doi:10.1007/s00158-010-0602-y.

[37] O. Sigmund, Morphology-based black and white filters for topology optimization, Structural and Multidisciplinary Optimization 33 (4) (2007) 401-424. doi :10.1007/s00158-006-0087-x.

[38] E. Andreassen, A. Clausen, M. Schevenels, B. Lazarov, O. Sigmund, Efficient topology optimization in matlab using 88 lines of code, Structural and Multidisciplinary Optimization 43 (1) (2011) 1-16. doi:10.1007/s00158-010-0594-7. 